\title{
COMPACTIFICATIONS OF $n$-SPACE BY AN ARC
}

\section{KYUNG WHAN KWUN ${ }^{1}$}

1. Introduction. We ask how many different spaces $X$ we obtain by compactifying $n$-space $R^{n}$ by an arc $A$. (We are not asking how many distinct pairs $(X, A)$ exist.) For $n$ large, most certainly uncountably many distinct $X$ ought to exist. However, if we require that $A$ be fitted nicely to $R^{n}$, the question becomes more interesting and perhaps harder. (On the other hand, if $X$ is required to be a topological manifold, then it must be a sphere in every dimension where the topological Poincaré conjecture is true.) We solve one aspect of this problem in the following form.

THEOREM. For each $n \geqq 4$, there exist uncountably many topologically distinct cohomology (or generalized) n-manifolds over integers $Z$ which are obtained from $R^{n}$ by compactifying by an arc.

By [6, Chapter IX], $X$ is necessarily $n$-sphere for $n=1$ and 2, the question is left open only for the case $n=3$. The proof makes a strong use of the method of [1] and constructions of [1] and [2]. As a corollary to $[1]$, we also obtain uncountably many generalized $n$-cells, $n \geqq 4$, which are AR but whose boundaries are not 1 -LC.

2. Contractible manifolds with boundary. Let $\Lambda$ denote the collection of all sequences of the form

$$
\left\{a_{1}\right\},\left\{a_{1}, a_{2}\right\},\left\{a_{1}, a_{2}, a_{3}\right\}, \cdots,\left\{a_{1}, a_{2}, \cdots, a_{k}\right\},\left\{a_{1}, \cdots\right\},
$$

where $a_{1}<a_{2}<a_{3}<\cdots$ are all positive integers. The $i$ th term of each $\lambda \in \Lambda$ will be denoted by $\lambda(i)$. We emphasize the following three properties of $\Lambda$ :

(1) For any integer $i$ and $\lambda \in \Lambda$ there exist infinitely many integers $i=i_{1}<i_{2}<\cdots$ such that $\lambda\left(i_{q}\right)=\lambda(i)$.

(2) If $\lambda, \lambda^{\prime} \in \Lambda$ and $\lambda \neq \lambda^{\prime}$, then there is a term in one of $\lambda$ and $\lambda^{\prime}$ which does not appear as terms in the other.

(3) The set $\Lambda$ is uncountable.

Let $n$ be a fixed integer greater than 3 . Let $M_{1}, M_{2}, \cdots$ be compact contractible $n$-manifolds such that $\pi_{1}\left(\operatorname{Bd} M_{i}\right)$ are pairwise nonisomorphic, each $\pi_{1}\left(\mathrm{Bd} M_{i}\right) \neq 1$ and no $\pi_{1}\left(\mathrm{Bd} M_{i}\right)$ is isomorphic to the free product of two nontrivial groups. The existence of such manifolds has been shown in [1], [2]. Further we assume that $M_{i} \times[0,1]$ is an

Received by the editors May 10, 1967.

${ }^{1}$ Research supported in part by NSF Grant GP 5868. 
$(n+1)$-cell. (See [1], [2].) For each $\lambda \in \Lambda$, consider the contractible $n$-manifolds

$$
M_{\lambda}=M_{\lambda(1)} \# M_{\lambda(2)} \# \cdots,
$$

the infinite connected sum as defined in [1].

3. Compactifications of $R^{n}$ by an arc. For each $\lambda \in \Lambda$, construct the space $X_{\lambda}$ which is obtained as follows. Let $N_{\lambda}$ be the one-point compactification of $M_{\lambda}$ by a point $p_{\lambda} . X_{\lambda} \subset N_{\lambda} \times[0,1]$ is defined to be the set

$$
\left[\left(\operatorname{Bd} M_{\lambda}\right) \cup\left\{p_{\lambda}\right\}\right] \times[0,1]=N_{\lambda} \times\{0,1\} .
$$

Proposition 1. $X_{\lambda}$ is a spherelike cohomology manifold over $Z$.

Proof. By [6], $\mathrm{Bd} M_{\lambda} \cup\left\{p_{\lambda}\right\}$ is a spherelike $(n-1)-\mathrm{cm}$. (cm stands for cohomology manifold over any principal ideal domain. Henceforth, the mention of the coefficient domains will be omitted.) Now if we take two copies of $M_{\lambda}$ and attach them along the boundaries, we obtain a space homeomorphic to $R^{n}$. (Recall $M_{i} \times[0,1]$ is an $(n+1)$-cell.) We write this fact as $2 M_{\lambda}=R^{n}$. Compactify $2 M_{\lambda}$ by the point $p_{\lambda}$. Then $\mathrm{Bd} M_{\lambda} \cup\left\{p_{\lambda}\right\} \subset n$-sphere which is the one-point compactification of $2 M_{\lambda}=R^{n}$. The closure of either complementary domain is homeomorphic to $N_{\lambda}$. By $[7$, Theorem 9.1, p. 312] or $\left[5\right.$, Theorem 2, p. 12] and [3, p. 434], $N_{\lambda}$ is a generalized $n$-cell in the sense of [7] and [3]. Consequently (see [3]), $N_{\lambda} \times[0,1]$ is a generalized $(n+1)$-cell and $X_{\lambda}=\operatorname{Bd}\left(N_{\lambda} \times[0,1]\right)$ is a spherelike $n$-cm.

Now let $A_{\lambda}$ be the arc in $X_{\lambda}$ corresponding to $p_{\lambda} \times[0,1]$.

Proposition 2. $X_{\lambda}-A_{\lambda}$ is homeomorphic to $R^{n}$.

Proof. Since $M_{i} \times[0,1]$ is an $(n+1)$-cell, $M_{\lambda} \times[0,1]$ is an infinite connected sum of $(n+1)$-cells and therefore is homeomorphic to $R^{n} \times[0,1)$. On the other hand $X_{\lambda}-A_{\lambda}$ is homeomorphic to $\mathrm{Bd}\left(N_{\lambda} \times[0,1]-A_{\lambda}\right)$. Hence it is homeomorphic to $\mathrm{Bd}\left(R^{n} \times[0,1)\right)$ $=R^{n} \times 0$.

In order to prove the theorem in the Introduction, it suffices to show that $X_{\lambda}$ and $X_{\lambda}^{\prime}$ are distinct if $\lambda \neq \lambda^{\prime}$.

4. Proof that $X_{\lambda}$ and $X_{\lambda}^{\prime}$ are not homeomorphic.

Proposition 3. $A_{\lambda} \subset X_{\lambda}$ is precisely the set of points of $X_{\lambda}$ at which $X_{\lambda}$ is not locally euclidean.

Proof. We observe that $N_{\lambda}$ is not locally 1 -connected at $p_{\lambda}$ in the sense of homotopy. Hence $X_{\lambda}$ is not locally 1 -connected at any in- 
terior point of $A_{\lambda}$. Hence $X_{\lambda}$ is not locally euclidean at any point of $A_{\lambda}$.

In view of Proposition 3, it suffices to show there is no homeomorphism $h$ of $\left(X_{\lambda^{\prime}}, A_{\lambda}^{\prime}\right)$ on to $\left(X_{\lambda}, A_{\lambda}\right)$.

Proposition 4. If $\lambda \neq \lambda^{\prime}$, there is no homeomorphism $h$ of $\left(X_{\lambda^{\prime}}, A_{\lambda^{\prime}}\right)$ onto $\left(X_{\lambda}, A_{\lambda}\right)$.

Proof. We use a simpler version of an argument in [1]. Because of a difference in situation (for instance, we do not have here group systems in the sense of [1]) we will reproduce a certain argument of [1] to show that such an argument in fact goes through in our present case. However, the reader's familiarity with [1] is assumed throughout. Suppose there exists such a homeomorphism $h$.

Let

$$
U_{i}=\operatorname{Bd} N_{\lambda}-\left(M_{\lambda(1)} \# M_{\lambda(2)} \# \cdots \# M_{\lambda(i-1)}\right) .
$$

Let $G_{i}$ be an abstract group isomorphic to $\pi_{1}\left(\mathrm{Bd} M_{\lambda(i)}\right)$. With suitably chosen base points, we may write

$$
\pi_{1}\left(U_{i}^{\prime}\right)=G_{i} * G_{i+1} * \cdots,
$$

the infinite free product, where $U_{i}^{\prime}=U_{i}-P_{\lambda}$. In order to avoid confusion, $G_{j} \subset \pi_{1}\left(U_{i}^{\prime}\right)$ will be denoted by $G_{j}^{i}$. Though it is impossible that all $\pi_{1}\left(U_{i}^{\prime}\right)$ have a common base point, it is nevertheless possible to have a homomorphism of $\pi_{1}\left(U_{i+1}^{\prime}, x_{i+1}\right) \rightarrow \pi_{1}\left(U_{i}^{\prime}, x_{i}\right)$ by $\pi_{1}\left(U_{i+1}^{\prime} X_{i+1}\right)$ $\rightarrow \pi_{1}\left(U_{i}^{\prime}, x_{i+1}\right) \rightarrow \pi_{1}\left(U_{i}^{\prime}, x_{i}\right)$ where the first homomorphism is induced by the inclusion and the second one by a path in $U_{i}^{\prime}$ connecting $x_{i}$ and $x_{i+1}$. See [1]. This way we define homomorphisms

$$
\pi_{1}\left(U_{i+1}^{\prime}\right) \rightarrow \pi_{1}\left(U_{i}^{\prime}\right)
$$

or more generally,

$$
\pi_{1}\left(U_{i+k}^{\prime}\right) \rightarrow \pi_{1}\left(U_{i}^{\prime}\right) .
$$

Under the latter homomorphism, $G_{j}^{i+\boldsymbol{k}}$ maps isomorphically onto $G_{j}^{i}$. See $[1$, p. 38].

Let $a=\left(p_{\lambda}, 1 / 2\right) \in A_{\lambda}$ and $P_{i}=U_{i} \times\left(1 / 2-1 / 2^{i}, 1 / 2+1 / 2^{i}\right)$. It is possible to identify

$$
\pi_{1}\left(P_{i}^{\prime}\right)=G_{i}^{i} * G_{i+1}^{i} * \cdots
$$

and define homomorphisms

$$
\pi_{1}\left(P_{i+k}^{\prime}\right) \rightarrow \pi_{1}\left(P_{i}^{\prime}\right),
$$


where $P_{j}^{\prime}=P_{j}-A_{\lambda}$ just as for $U_{i}^{\prime} s$. Let $b \in A_{\lambda}^{\prime}$ be such that $h(b)=a$ and $Q_{1} Q_{2} \cdots$ be a sequence of neighborhoods of $b$ having the same relation to $b$ as $P_{1}, P_{2}, \cdots$ do to $a$.

Let $\quad Q_{j}^{\prime}=h\left(Q_{j}\right)-A_{\lambda}$. Then $\pi_{1}\left(Q_{j}^{\prime}\right)=F_{j} * F_{j+1} * \cdots, \quad$ where $F_{k} \simeq \pi_{1}\left(\mathrm{Bd} M_{\lambda(k)}^{\prime}\right)$. Find integers $j, k, s, t$ such that $P_{i}^{\prime} \supset Q_{s}^{\prime} \supset P_{j}^{\prime}$ $\supset Q_{\imath}^{\prime} \supset P_{k}^{\prime}$. As in [1], it is possible to find homomorphisms

$$
\begin{aligned}
\pi_{1}\left(P_{k}^{\prime}\right)=G_{k}^{k} * G_{k+1}^{k} * \cdots \\
\\
\downarrow f \\
\pi_{1}\left(Q_{t}^{\prime}\right)=F_{t}^{t} * F_{t+1}^{t} * \cdots \\
\downarrow g \\
\pi_{1}\left(P_{j}^{\prime}\right)=G_{i}^{i} * G_{i+1}^{i} * \cdots \\
\downarrow f^{\prime} \\
\pi_{1}\left(Q_{s}^{\prime}\right)=F_{s}^{s} * F_{s+1}^{s} * \cdots
\end{aligned}
$$

such that $g f$ is the monomorphism we defined above up to a conjugacy automorphism of $\pi_{1}\left(P_{j}^{\prime}\right)$ and $f^{\prime} g$ is the monomorphism similarly definable for $Q^{\prime} s$ up to a conjugacy automorphism of $\pi_{1}\left(Q^{\prime} s\right)$.

Without loss of generality, we may suppose that $G_{k}^{k}$ is not isomorphic to any $F_{m}$. The bar above homomorphisms will denote appropriate restrictions. Since $G_{k}^{k}$ is not a free product of two nontrivial groups, by Kurosh's subgroup theorem [3], $f\left(G_{k}^{k}\right)$ is a conjugate of some subgroup of some $F_{m}^{t}$. Then since $g f\left(G_{\boldsymbol{k}}^{k}\right)$ is a conjugate of $G_{k}^{i}, g\left(F_{m}^{t}\right)$ is a conjugate of $G_{\boldsymbol{k}}^{i}$ we have

$$
G_{k}^{k} \stackrel{\bar{f}}{\rightarrow} \text { conjugate of } F_{m}^{t} \stackrel{\bar{g}}{\rightarrow} \text { conjugate of } G_{k}^{i} .
$$

Since $\bar{f}, \bar{g}$ are monomorphisms and $\bar{g} \bar{f}$ is an isomorphism, $\bar{f}$ is an isomorphism. Thus $G_{k}^{k} \simeq F_{m}$, a contradiction.

5. Some generalized $n$-cells. Consider $N_{\lambda} \times[0,1]$. If we shrink $A_{\lambda}$ to a point, we obtain an $(n+1)$-cell. In this $(n+1)$-cell, the subset corresponding to $N_{\lambda} \times 0$ sits as a strong deformation retract. Hence $N_{\lambda}$ is an AR (absolute retract). This leads to

COROLlARy. For each $n \geqq 4$, there exist uncountably many generalized $n$-cells $N_{\lambda}$ which are $A R$ but whose boundaries are not 1-LC.

REMARK. $N_{\lambda}$ is never a cartesian factor of a cell. If $N_{\lambda} \times C$ is a cell, Bd $N_{\lambda} \times$ Int $C$ would be an open subset of a sphere and Bd $N_{\lambda}$ would be locally contractible. 


\section{REFERENCES}

1. M. L. Curtis and K. W. Kwun, Infinite sums of manifolds, Topology 3 (1965), 31-42.

2. L. C. Glaser, Uncountably many contractible open 4-manifolds, Topology 6 (1967), 37-42.

3. K. W. Kwun and Frank Raymond, Factors of cubes, Amer. J. Math. 84 (1962), 433-440.

4. A. G. Kurosh, The theory of groups, Vol. II, Chelsea, New York, 1956.

5. Frank Raymond, Separation and union theorems for generalized manifolds with boundary, Michigan Math. J. 7 (1960), 7-21.

6. - The end point compactification of manifolds, Pacific J. Math. 10 (1960), 947-963.

7. R. L. Wilder, Topology of manifolds, Amer. Math. Soc. Colloq. Publ. Vol. 32, Amer. Math. Soc., Providence, R. I., 1949.

Michigan State University 